\title{
Gender Differences in Neuropsychiatric Symptoms in Mild to Moderate Alzheimer's Disease Patients Undergoing Switch of Cholinesterase Inhibitors: A Post Hoc Analysis of the EVOLUTION Study
}

\author{
Delia Colombo, MD, Carlo Caltagirone, $\mathrm{MD}^{2,3}$ Alessandro Padovani, $\mathrm{PhD}^{4}$, \\ Sandro Sorbi, MD, ${ }^{5,6}$ Gianfranco Spalletta, PhD, Lucia Simoni, PhD, \\ Alessandra Ori, MD, and Emanuela Zagni, $\mathrm{MSc}^{1}$
}

\begin{abstract}
Background: Alzheimer's disease (AD) is characterized by progressive cognitive decline, often associated with Behavioral and Psychological Symptoms of Dementia (BPSD). Acetylcholinesterase inhibitors (ChEi) may attenuate cognitive decline and mitigate BPSD. The EVOLUTION group found that the switch from oral ChEi to transdermal rivastigmine patch formulation resulted in improvement/stabilization in the frequency of clinically relevant BPSD, but gender-specific subgroup analyses were not reported.

Methods: Participants underwent Neuropsychiatric Inventory to assess the frequency and severity of neuropsychiatric symptoms at baseline and 3 and 6 months after the switch from oral ChEi to transdermal rivastigmine patch. A descriptive post hoc analysis was conducted to assess whether there were gender-based differences in BPSD profile during the 6 months after the switch.

Results: The entire sample consisted of 475 patients, 274 women and $201 \mathrm{men}$. Women were on average slightly older and with poorer cognitive performance $(60.6 \%$ of the women had moderate-AD, defined as Mini-Mental State Examination [MMSE] score of 10-17, vs. 43.8\% of men). In mild-AD patients (MMSE score 18-26), the frequency of BPSD did not change significantly over time and an association was found between gender and depression (odds ratio; OR [95\% confidence interval; $\mathrm{CI}]$ female vs. male $=3.32$ [1.44-7.67]), anxiety (2.42 [1.23-4.79]), apathy (2.25 [1.07-4.70]), nighttime behavior disturbances (3.97 [1.66-9.49]), and appetite/eating abnormalities (2.39 [1.10-5.18]). Moderate-AD female patients had euphoria more frequently than male patients (OR [95\% CI] female vs. male $=3.67[1.25-10.74]$ ). The frequency of delusions, anxiety, and irritability decreased during the first 3 months after the switch, independently of gender.

Conclusion: Mild-AD women tended to suffer more frequently from BPSD than men; in the 3 months after treatment switch, moderate-AD patients showed a decrease in delusions, anxiety, and irritability, with no significant differences between genders. Ad hoc studies to investigate this potential gender effect in AD could be well worthwhile.
\end{abstract}

Keywords: gender, Alzheimer's disease, cholinesterase inhibitors, Behavioral and Psychological Symptoms of Dementia (BPSD)

\footnotetext{
${ }^{1}$ Patient Access, Novartis Farma S.p.A., Origgio, Italy.

${ }^{2}$ Neuropsychiatry Laboratory, Department of Clinical and Behavioral Neurology, IRCCS Santa Lucia Foundation, Rome, Italy.

${ }^{3}$ Department of System Medicine, University of Rome "Tor Vergata," Rome, Italy.

${ }^{4}$ Department of Clinical and Experimental Sciences, University of Brescia, Brescia, Italy.

${ }^{5}$ Department of Neuroscience, Psychology, Drug Research and Child Health, University of Florence, Florence, Italy.

${ }^{6}$ IRCCS Don Gnocchi, Florence, Italy.

${ }^{7}$ MediNeos Observational Research, Modena, Italy.
}

(C) Delia Colombo et al. 2018; Published by Mary Ann Liebert, Inc. This Open Access article is distributed under the terms of the Creative Commons License (http://creativecommons.org/licenses/by/4.0), which permits unrestricted use, distribution, and reproduction in any medium, provided the original work is properly cited. 


\section{Introduction}

A LZHEIMER'S DISEASE (AD) is an insidious illness characterized by the progressive decline of mental function; in addition to cognitive deficiencies, $\sim 90 \%$ of patients at any given point in the course of their illness exhibit various $\mathrm{Be}$ havioral and Psychological Symptoms of Dementia (BPSD). ${ }^{1}$ BPSD often occur in clusters: hyperactivity, including agitation and aggression; affective symptoms, including depression and anxiety; or psychosis, including both delusions and hallucinations. ${ }^{2}$ These symptoms are associated with poorer quality of life, ${ }^{3}$ a heavier burden on caregivers, ${ }^{3,4}$ increased risk of institutionalization, ${ }^{5,6}$ and increased mortality.

Genders differ: women with AD suffer delusions and hallucinations more often ${ }^{8,9}$ and earlier ${ }^{10}$ than men, and depression too is more prevalent in women with AD. ${ }^{9,11}$

Moreover, men more commonly display apathy, ${ }^{12}$ aggression, ${ }^{12,13}$ and vegetative changes. ${ }^{12}$

Appropriate, tailored interventions are essential to provide quality care. Such interventions include both nonpharmacological strategies and psychotropic agents. The etiology of BPSD has not been clearly delineated, but studies assessing the benefits of acetylcholinesterase inhibitors (ChEi) on BPSD suggest that some of these symptoms, such as agitation, apathy, and psychosis, may reflect a specific central cholinergic deficiency syndrome. ${ }^{14} \mathrm{~A}$ meta-analysis found that 3-12 months of treatment with ChEi had a modest effect on global BPSD scores. ${ }^{15}$ The data on the efficacy of rivastigmine in moderating BPSD in AD patients are variable. In one 6month study, changes in Neuropsychiatric Inventory (NPI) scores were no different between the rivastigmine and placebo groups. ${ }^{16}$ In a different, 12-month study, agitation/aggression and anxiety/phobias improved significantly among AD patients treated with rivastigmine. ${ }^{17}$

Norton et al. have suggested that caregiver interventions may become an important complement to pharmacological and other approaches to slowing the progression of dementia; they showed that for adult caregivers who are children of the patient, the personality traits of the caregivers are associated with the rate of cognitive decline in the receivers (CRs) with $\mathrm{AD}$, regardless of coresidency. ${ }^{18}$

Furthermore, closeness and loss of closeness in the caregiver-CR dyad may be associated with either positive or adverse outcomes for caregivers. ${ }^{19}$

The EVOLUTION observational study evaluated the switch in ChEi in a sample of 635 AD patients who were no longer responsive to the initial treatment, with reduced tolerance and/or compliance and the loss of at least two points on their Mini-Mental State Examination (MMSE) score in the last 6 months. In analysis of a subsample of 423 patients in the EVOLUTION study, the study group found that the switch from oral $\mathrm{ChEi}$ to transdermal rivastigmine patch formulation not only slowed the progression of global cognitive impairment but also resulted in improvement/stabilization in the frequency of clinically relevant BPSD. ${ }^{20}$ While men and women are known to differ in the risk, pathogenesis, clinical manifestations, and prognosis of $\mathrm{AD},{ }^{21-23}$ the EVOLUTION study Group did not report gender-specific subgroup analyses, and current clinical guidelines do not differentiate between men and women in the use of ChEi. Accordingly, for the sample of patients in the EVOLUTION study, we conducted a post hoc analysis to assess whether there were gender-based differences in BPSD profile during 6 months after the switch from oral ChEi to transdermal rivastigmine patch.

\section{Methods}

This post hoc analysis is part of the gender medicine project MetaGeM, designed to describe and analyze clinical outcomes, therapeutic approaches and safety data according to gender, based on nine observational studies; the MetaGeM methodology has been described extensively elsewhere. ${ }^{24}$

The EVOLUTION study was conducted in 38 outpatient memory clinics across Italy, on subjects who satisfied the following criteria: (1) diagnosis of probable $\mathrm{AD}$ according to the criteria of the National Institute of Neurological and Communicative Disorders and Stroke and the Alzheimer's Disease and Related Disorders Association (NINCDSADRDA $)^{25}$; (2) mild to moderate severity of dementia, defined as $\mathrm{MMSE}^{26}$ score of 26 to 10 ; (3) onset of symptoms at least 6 months before inclusion in the sample; (4) treatment with ChEi for at least 6 months, and first switch to another type of ChEi therapy due to lack of response and/or reduced compliance (i.e., lack or loss of efficacy, defined as a decline of at least two points in the MMSE score in the last 6 months) and/or reduced tolerance (due to side effects or failure to follow recommended oral dosing regimen); (5) vision and hearing sufficient for compliance with testing procedures; and (6) presence of caregiver able to understand all testing procedures. Hospitalized patients were excluded.

The EVOLUTION study design and methodology are described elsewhere. ${ }^{20}$ This post hoc analysis used a subsample of EVOLUTION patients: patients without psychiatric disorders, who switched from oral ChEi to rivastigmine transdermal patch at baseline and who underwent 3- and 6month follow-up visits. The switch followed treatment guidelines for $\mathrm{AD}$; in particular, the practice for switching called for an immediate switch from other ChEi to patch $4.6 \mathrm{mg} / 24$ hours, and 1 month after the switch the dose was increased to $9.5 \mathrm{mg} / 24$ hours. Patients were then kept on this dosage unless they experienced adverse events; in this case, the rivastigmine patch dosage was reduced to $4.6 \mathrm{mg} /$ 24 hours.

Women and men were described and compared at baseline according to sociodemographic variables (age, education, marital and occupational status, smoking habits, and alcohol consumption) and clinical features (concomitant diseases, time to onset of symptoms and to diagnosis, ChEi therapy in the 6 months before baseline). Caregivers (who remained the same for the entire length of the study) were described at baseline (age, gender, type, and cohabitation with patient) and their characteristics compared between male and female patients.

Clinical data collection included MMSE (adjusted by age and educational level for elderly patients ${ }^{27}$ ) and NPI for the assessment of BPSD, carried out at baseline and at 3- and 6month follow-up.

The NPI is a useful instrument for characterizing the psychopathology of dementia syndromes, investigating the neurobiology of brain disorders with neuropsychiatric manifestations, distinguishing among different syndromes and assessing the efficacy of treatment. ${ }^{28}$ The validity and 
reliability of the Italian version of the NPI have been demonstrated. ${ }^{29}$ The NPI was used to assess the frequency and severity of neuropsychiatric symptoms in 12 domains: delusions, hallucinations, agitation, depression, anxiety, euphoria, apathy, disinhibition, irritability, aberrant motor behavior, nighttime behavior disturbances, and appetite and eating abnormalities. ${ }^{24}$ The severity and frequency of each symptom were scored on the basis of structured questions administered to the caregiver. Frequency was rated from 1 (only occasional) to 4 (very frequent) and severity from 1 (mild) to 3 (severe). If the symptom was absent, the score was 0 . A composite score for each symptom was calculated, namely the product of the frequency and severity scores, with range accordingly from 0 to 12 .

A first exploratory analysis was conducted, considering for each domain patients with available NPI scores at baseline and 3- and 6-month follow-up and comparing women and men at each study visit with regard to the presence of neuropsychiatric symptoms. BPSD were considered present for composite scores $\geq 1$. A composite score $\geq 4$ indicated the presence of a clinically relevant symptom typically associated with therapeutic intervention; a score of 1-3, mild symptoms usually not requiring specific treatment; and a score of 0 , no symptom. ${ }^{30,31}$ For homogeneous gender comparison, the sample was stratified according to severity of dementia: mild-AD patients (i.e., an MMSE score of 26-18) and moderate-AD patients (MMSE score of 17-10).

Comparisons by gender were performed by Student's $t$ test (for continuous variables) and $\chi^{2}$ or Fisher's exact test (for categorical variables). For the post hoc analyses, the significance threshold was set at 0.05 (all $p$ values presented are exploratory, so no correction for multiple testing was applied).

Confirmatory logistic regressions for repeated measures were then run to get a further evaluation of the association between gender and BPSD. A model for each BPSD domain was estimated. As binary dependent variable, the presence (i.e., a NPI composite score $\geq 1$ )/absence of each specific BPSD at time $x$ ( $x=$ baseline, 3-month follow-up, 6-month follow-up) was considered and gender was introduced in the models as constant effect over time (no interaction between time and gender was hypothesized).

The models were then adjusted for age $(\leq 60,60-70,70-80$, $>80$ years), marital status (married/cohabiting, single, divorced/separated, widowed), education (low [none, primary], medium [junior high school], high [high school, university/ postgraduate]), smoking (smoker, exsmoker, nonsmoker), alcohol consumption (none, moderate to high), concomitant ischemic heart disease, hypertension, dyslipidemia, diabetes, cerebrovascular disease (yes or no), caregiver's gender, caregiver's relationship to patient (son/daughter; husband/ wife; other), ongoing antipsychotic and antidepressant therapy (yes or no), and previous treatment with oral rivastigmine (yes or no). The ongoing antipsychotic and antidepressant therapies were considered time-dependent variables and so evaluated at each time point. All the other covariates were assessed at baseline visit and considered constant over time. The results of the multivariate regressions provided an estimate of the association between gender and the presence of BPSD, adjusted for potential confounding factors.

We were also interested in estimating the effect of time on the presence of BPSD in mild- and moderate-AD patients, holding gender and all the other variables of each model constant. For this reason, the visit (baseline, 3-month followup, 6-month follow-up) was also included as covariate in the model.

To control for MMSE that differed significantly between genders, the models were estimated in two different subpopulations, mild- and moderate-AD patients.

Finally, we compared the results of these models with those of an exploratory analysis in which the proportion of patients with NPI remission (i.e., symptoms present at baseline and absent at follow-up) was calculated and compared by gender.

Patients with missing data in some parameters were not excluded from the analysis, but only from the analysis of those parameters.

All analyses were performed with SAS v9.4 and Enterprise Guide v7.1.

\section{Results}

\section{Baseline demographics, lifestyle data,} and clinical characteristics

The sample analyzed consisted of a total of 475 patients: 274 women $(57.7 \%)$ and 201 men $(42.3 \%)$; baseline demographic data are provided in Table 1. The women were slightly older (mean \pm standard deviation [SD] $77.0 \pm 7.0 \mathrm{vs.}$ $75.5 \pm 7.0$ in men, $p=0.022$ ), less likely to be married/cohabiting $(48.7 \%$ vs. $85.6 \%$ of men, $p$-value $<0.001)$, and less well educated $(71.2 \%$ with at most primary school vs. $61.2 \%$ of men, $p=0.010)$. Men were more likely to be past smokers ( $43.5 \%$ vs. $8.1 \%$ of women) or current smokers $(15.5 \%$ vs. $9.6 \%$ of women, $p<0.001)$ and to be frequent alcohol consumers $(38.5 \%$ vs. $15.9 \%$ of women, $p<0.001)$. No gender differences were found in employment status or concomitant diseases (except ischemic heart disease, more frequent in men [22.4\%] than in women [12.4\%], $p=0.004)$.

There was no significant difference between men and women in time to onset of symptoms and to diagnosis: the means $( \pm \mathrm{SD})$ for these variables were $1.3( \pm 1.1)$ and 2.4 $( \pm 1.7)$ years for men and $\pm 1.4( \pm 2.2)$ and $2.7( \pm 2.0)$ years for women ( $t$-test $p$-values, male vs. female $=0.275$ and 0.159 , respectively).

In the 6 months before baseline, 162, 14, and 25 men $(80.6 \%, 7.0 \%, 12.4 \%$, respectively) were treated with donepezil, galantamine, and rivastigmine (oral formulation) and 235,24 , and 15 women $(85.8 \%, 8.8 \%$, and $5.5 \%)$ were treated with donepezil, galantamine, and rivastigmine (oral formulation), respectively ( $\chi^{2}$ test $p$-value, ChEi treatment male vs. female $=0.023$ ). The daily dosage of ChEi treatment before baseline did not differ between males and females.

The reasons for the switch in ChEi therapy at baseline did not differ substantially between male and female patients (Fig. 1).

Among women, $5.5 \%$ were treated at baseline with memantine versus $3.6 \%$ of men ( $\chi^{2}$ test $p$-value, male vs. female $=0.340$ ). Women more frequently took antidepressants (sertraline, escitalopram, citalopram, venlafaxine, paroxetine) $\left(15.3 \%\right.$ vs. $7.5 \%$ of men; $\chi^{2}$ test $p$-value $\left.=0.009\right)$, while no substantial gender differences were found at baseline in antipsychotic treatment (risperidone, olanzapine, quetiapine, ziprasidone, aripiprazole, haloperidol) $-5.1 \%$ of women versus $8.5 \%$ of men; $\chi^{2}$ test $p=0.144$. 
Table 1. Baseline Demographic, Lifestyle, and Clinical Data of MetaGem EVOLUTiOn Study Population

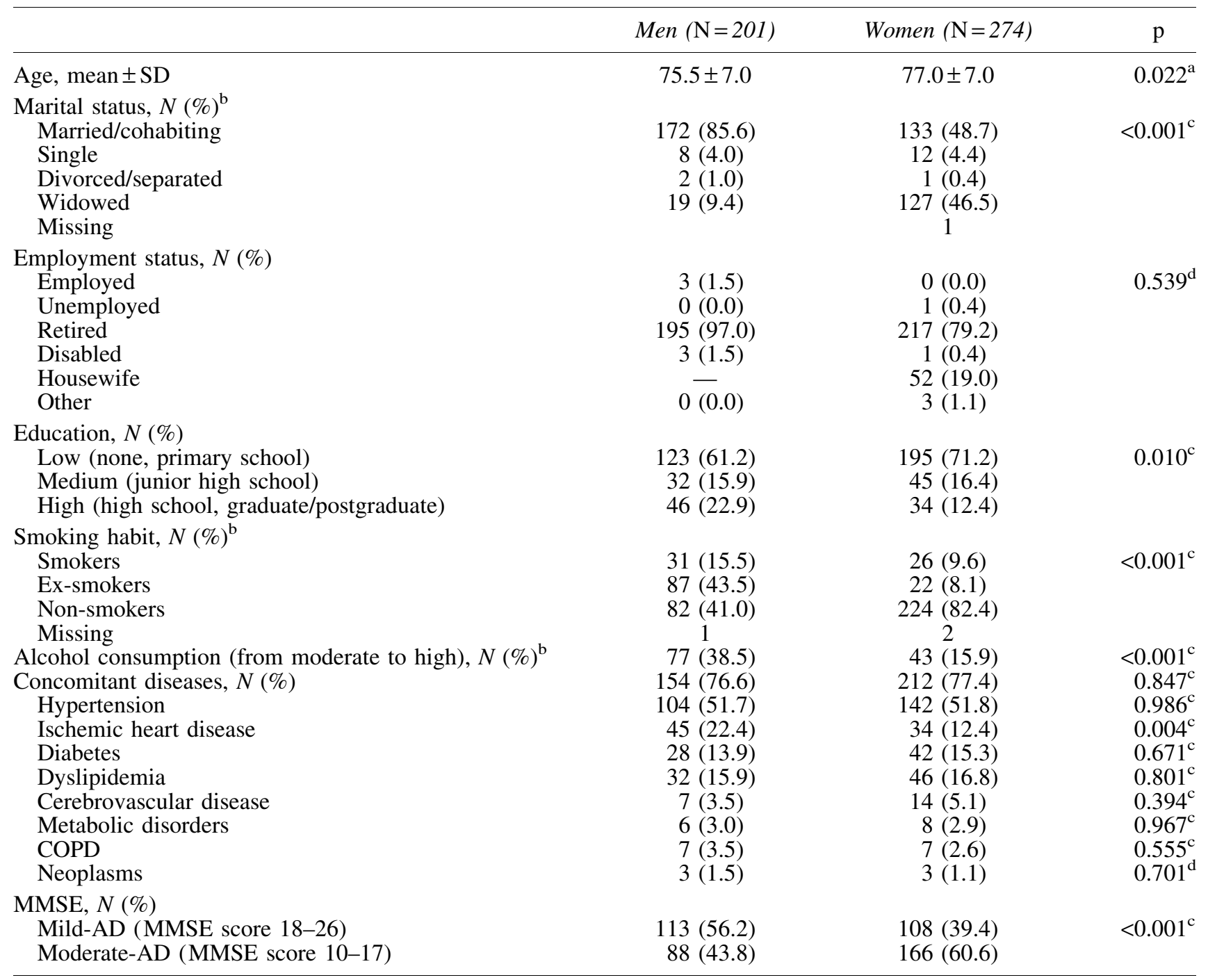

${ }^{\mathrm{a}} t$-Test.

${ }^{\mathrm{b}}$ Percentages computed over non missing responses.

${ }^{\mathrm{c}}$ Chi-squared test.

For marital status, the following classes were considered: married/cohabiting versus single, divorced/separated, widowed. For alcohol consumption, the following classes were considered: no alcohol consumption, from moderate to high.

${ }^{\mathrm{d}}$ Two-sided Fisher's exact test.

For employment status, the following classes were considered: housewife/retired, other.

AD, Alzheimer's disease; COPD, chronic obstructive pulmonary disease; MMSE, Mini-Mental State Examination; SD, standard deviation.

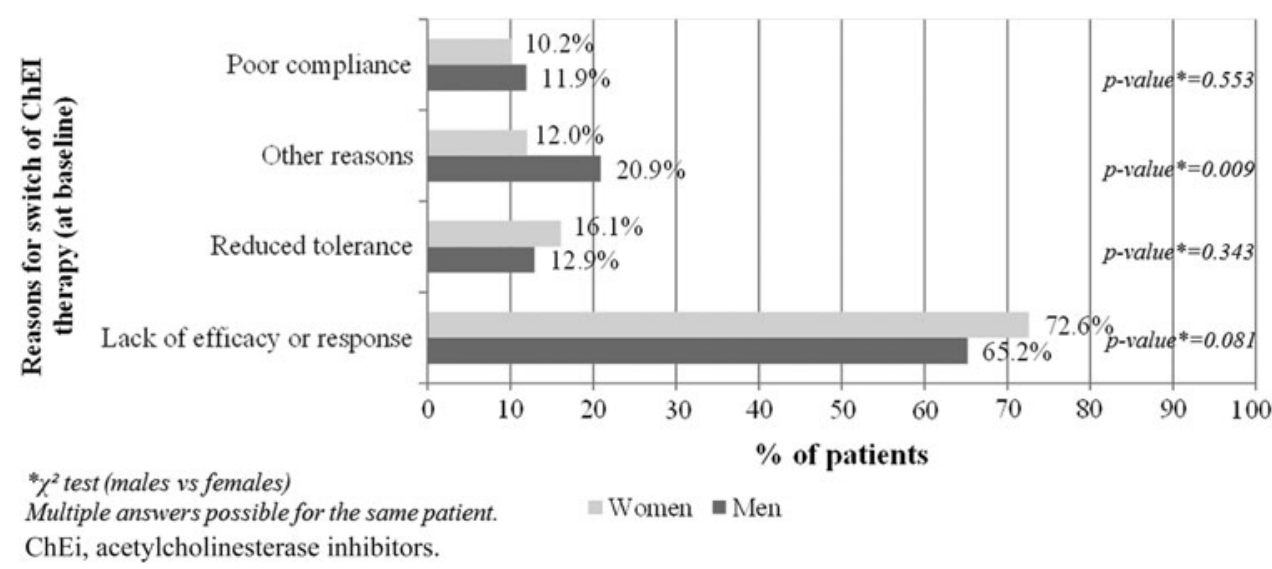

FIG. 1. Reasons for switch of ChEi therapy at baseline by gender. 
FIG. 2. MMSE score at study visits by gender.

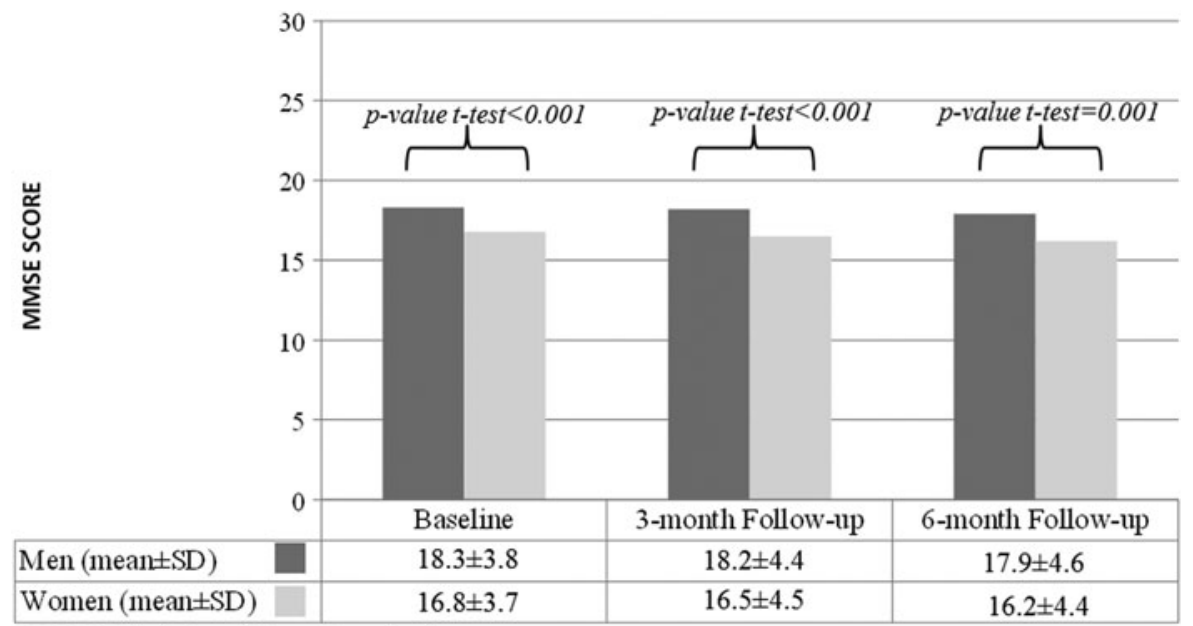

MMSE, Mini-Mental State Examination.
As shown in Table 1, 113 men (56.2\%) and 108 women (39.4\%) were mild-AD at baseline (MMSE score 18-26); accordingly, $43.8 \%$ of the men and $60.6 \%$ of the women were moderate-AD (MMSE score $10-17)\left(\chi^{2}\right.$ test $p$-value, MMSE males vs. females $<0.001)$. At each point of measurement, women's MMSE score was significantly lower than men's (Fig. 2).

Men had a female caregiver in $89.6 \%$ of the cases versus $60.9 \%$ for female patients ( $\chi^{2}$ test $p$-value $\leq 0.001$ ); for 131 male patients $(65.8 \%)$, the caregiver was the wife, while 165 female patients $(61.6 \%)$ had a son or daughter as caregiver and 46 $(17.2 \%)$ had other caregivers (brother/sister, daughter-/son-inlaw, nephew/niece, brother-/sister-in-law, friend) $\left(\chi^{2}\right.$ test type of caregiver, male vs. female patients, $p$-value $<0.001)$. Other caregiver characteristics are shown in Table 2.

\section{$B P S D$ profile in women and men}

Figures 3 and 4 show the results of the exploratory analysis of frequency of NPI symptoms at study visits in the entire

Table 2. Caregiver Characteristics at Baseline

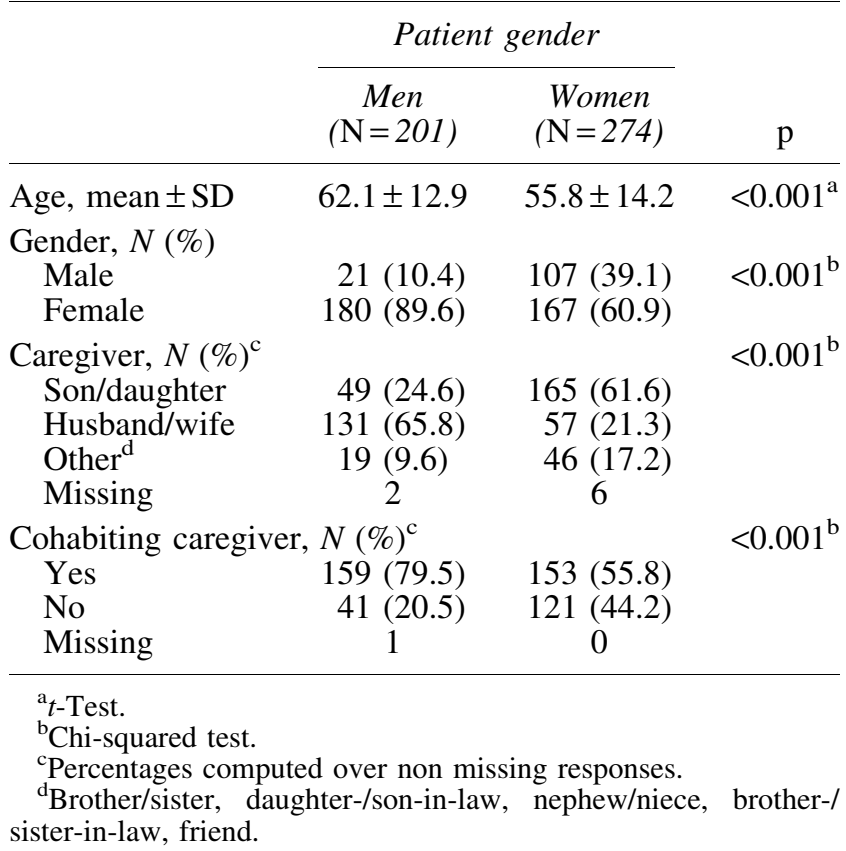

sample and stratified by severity of symptoms (mild-AD and moderate-AD patients). The patients with missing NPI scores (not included in the analyses) did not differ significantly from those without missing scores in age, gender, or MMSE score at baseline ( $p$-value $t$-test and $\chi^{2}$ test patients with vs. without score $>0.05$, data not shown).

\section{BPSD profile in women and men at baseline}

Entire sample. The NPI assessment showed a higher frequency of delusions, hallucinations, depression, anxiety, apathy, and aberrant motor behavior at baseline in women than in men (see Figs. 3 and 4). No gender differences emerged in the other symptoms at baseline.

Breakdown between mild-AD and moderate-AD patients. For mild-AD patients, at baseline, the following symptoms were more frequent in women than men: hallucinations $(15.6 \%$ vs. $5.7 \%, p$-value $=0.021)$, depression $(58.6 \%$ vs. $32.1 \%, p$-value $<0.001)$, anxiety $(53.5 \%$ vs. $27.8 \%, p$-value $<0.001)$, apathy $(69.4 \%$ vs. $51.8 \%, p$-value $=0.010)$, nighttime behavior disturbances (38.7\% vs. $20.4 \%, p$-value $=0.006)$, and appetite/eating abnormalities $(34.0 \%$ vs. $18.0 \%, p$ value $=0.011)($ see Figs. 3 and 4$)$.

For moderate-AD patients, euphoria was more frequent at baseline in women $(11.3 \%)$ than in men $(2.7 \%, p$-value $=$ $0.028)$; differently from mild-AD patients, in moderate-AD patients nighttime behavior disturbances were more frequent at baseline in men than in women $(37.7 \%$ of men vs. $24.5 \%$ of women, $p$-value $=0.038$ ). No gender differences were observed for the other symptoms (see Figs. 3 and 4).

\section{BPSD profile in women and men \\ at 3- and 6-month follow-up}

Entire sample. The NPI assessment showed a higher frequency of delusions and depression in women than men at all points of study; anxiety too was more frequent in women than in men at 3- and at 6-month follow-up (see Fig. 3), as was irritability at 6-month follow-up (see Fig. 4).

No gender differences in the other symptoms were found at follow-up.

Breakdown between mild-AD and moderate-AD patients. At 3-month follow-up, mildly demented women had 


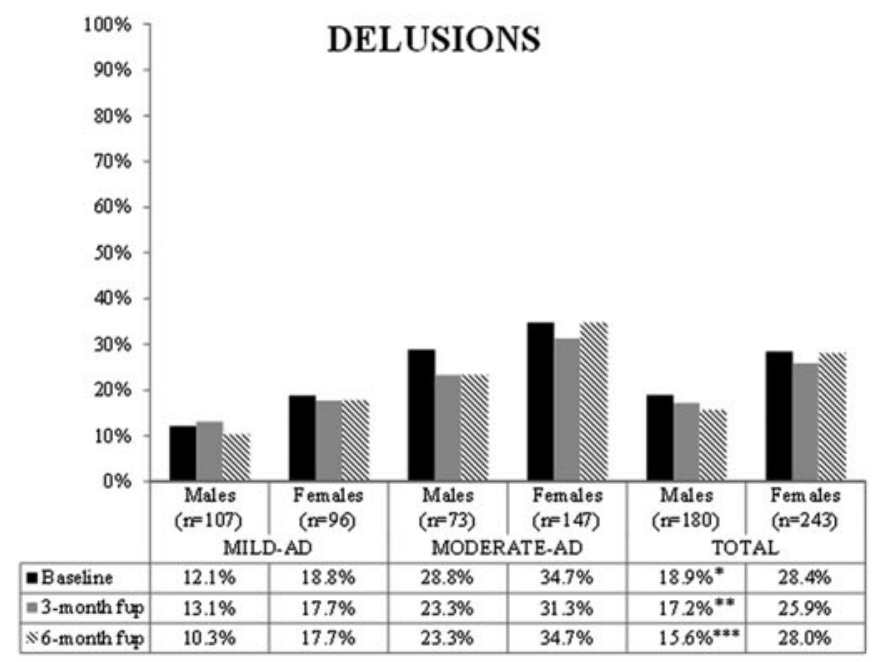

${ }^{*} X^{2} p$-value $(m$ vs $f)=0.024 ;{ }^{* *} X^{2} p$-value $(m$ vs $f)=0.033 ;{ }^{* * *} X^{2} p$-value $(m$ vs $f)=0.003$.

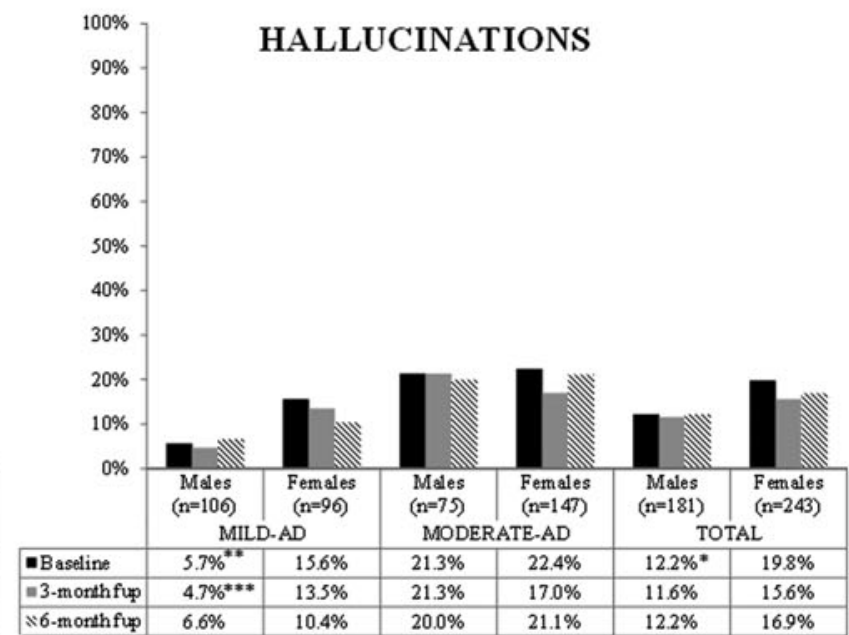

${ }^{*} X^{2} p$-value $(m$ vs $f)=0.037 ;{ }^{* *} X^{2} p$-value $(m v s f)=0.021 ;{ }^{* * *} X^{2} p$-value $(m$ vs $f)=0.028$.
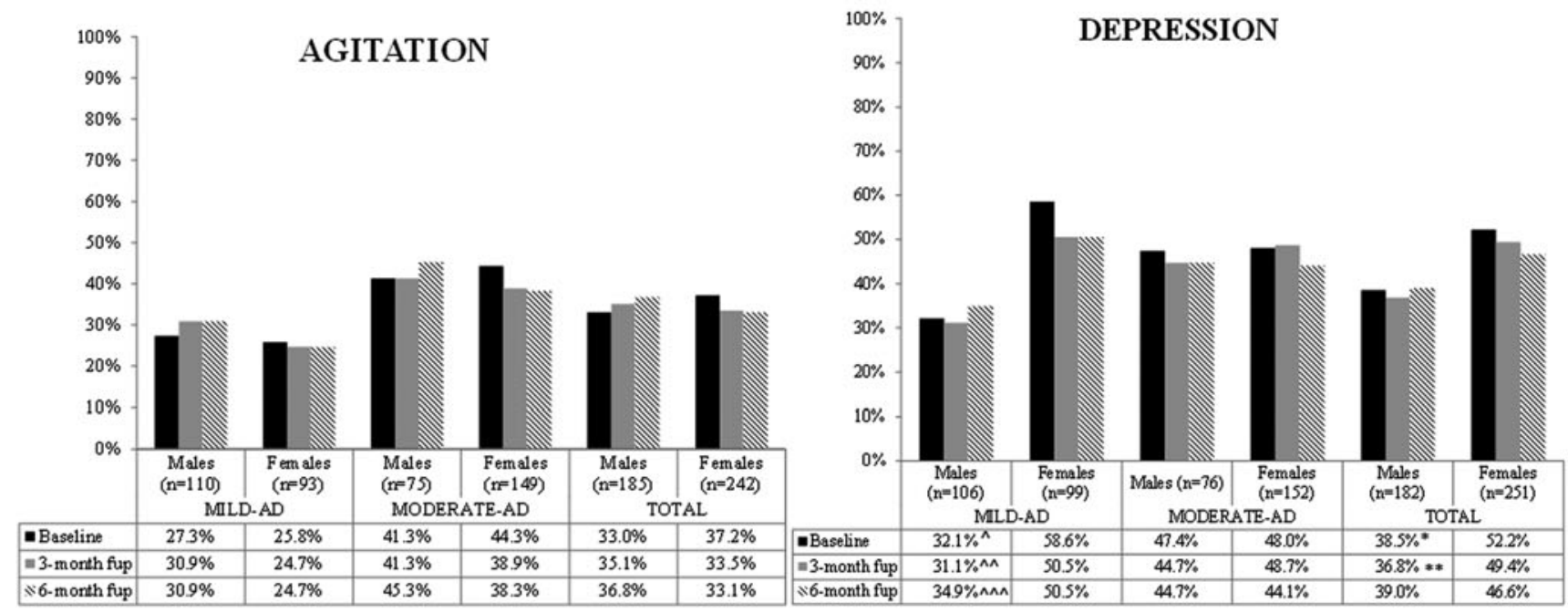

${ }^{*} X^{2} p$-value $(m$ vs $f)=0.005 ;{ }^{* *} X^{2} p$-value $(m$ vs $f)=0.009$

${ }^{\wedge} X^{2} p$-value $(m$ vs $f)<0.001 ;{ }^{\wedge} X^{2} p$-value $(m$ vs $f)=0.005 ; M^{2} p$-value $(m$ vs $f)=0.024$.

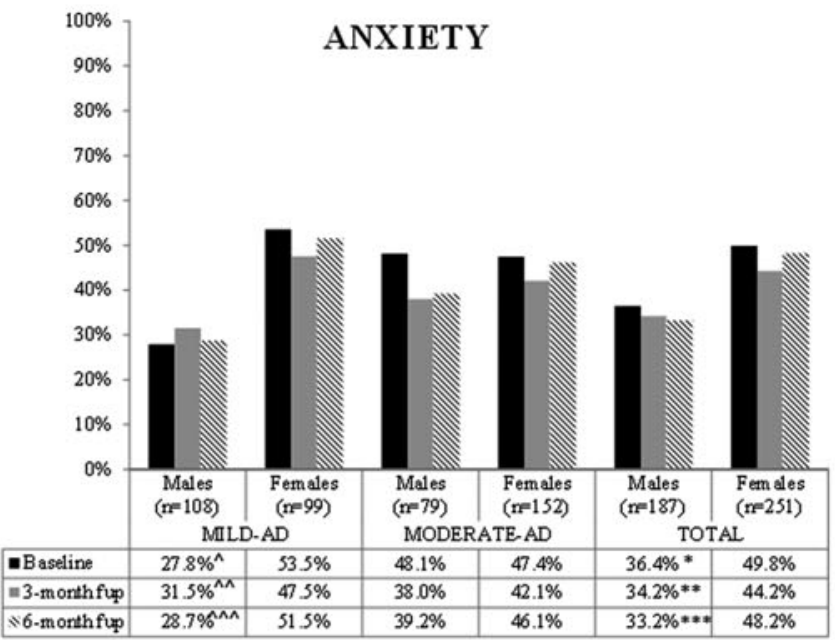

${ }^{*} X^{2} p$-value $(m$ v $f)=0.005 ; * * X{ }^{2} p$-value $(m$ vs $f)=0.035 ; * * * X^{2} p$-value $(m$ v $f f)=0.002$; ${ }^{\wedge} X^{2} p$-value $(m$ vs $f)<0.001 ;{ }^{M} X^{2} p$-value $(m v s f)=0.019 ;{ }^{M} X^{2} p$-value $(m$ vs $f)<0.001$.

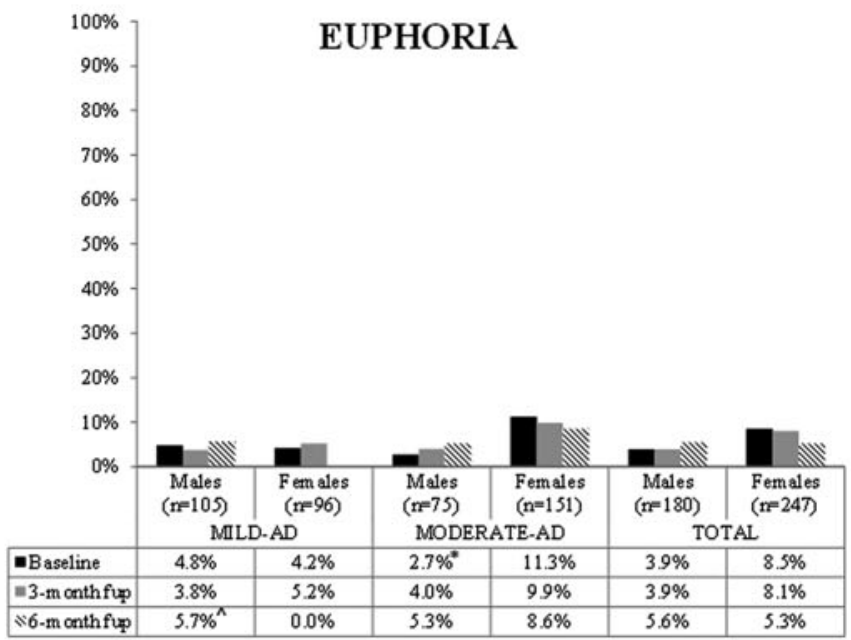

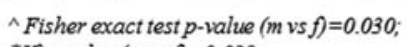

${ }^{*} X^{2} p$-value $(m v s f)=0.028$.

Not significant $(>0.05)$ p-values werenot reported NPI, Neuropsychiatric Inventory.

FIG. 3. Frequency of neuropsychiatric symptoms according to NPI at study visits by gender-delusions, hallucinations, agitation, depression, anxiety and euphoria. 


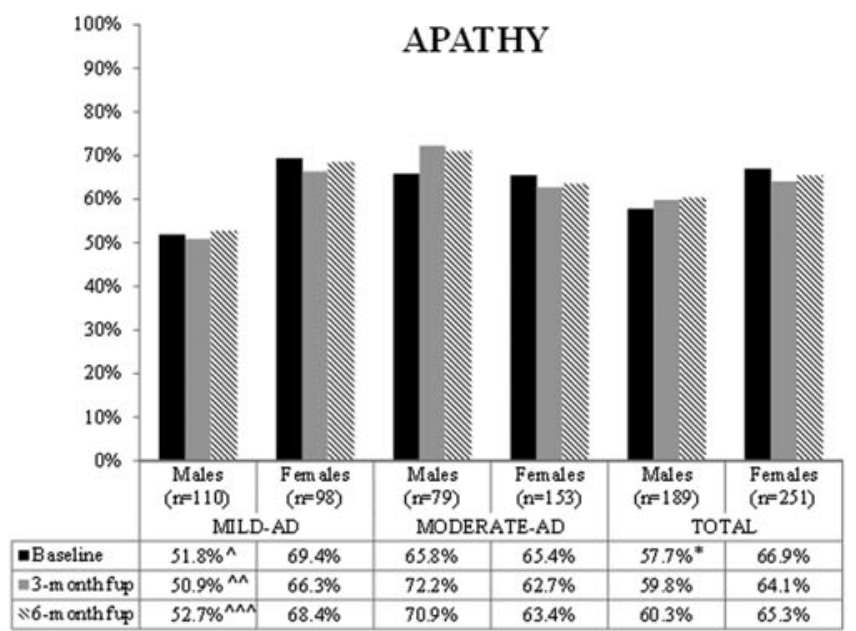

${ }^{*} X^{2} p$-value $(m$ vs $f)=0.047$

${ }^{\wedge} X^{2} p$-value $(m$ vs $f)=0.010 ;{ }^{M} X^{2} p$-value $(m v s f)=0.024 ;{ }^{M} X^{2} p$-value $(m$ vs $f)=0.022$.

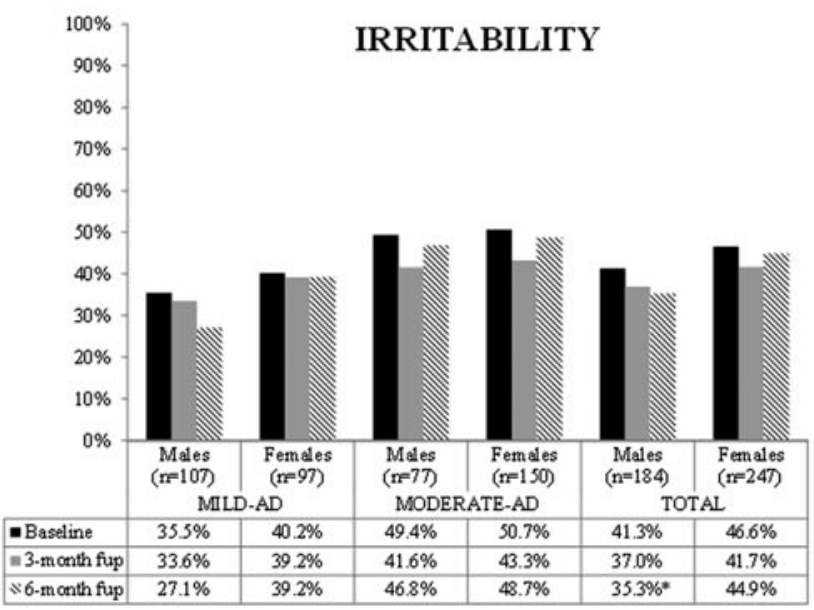

${ }^{2} X^{2} p$-value $(m v s f)=0.045$.

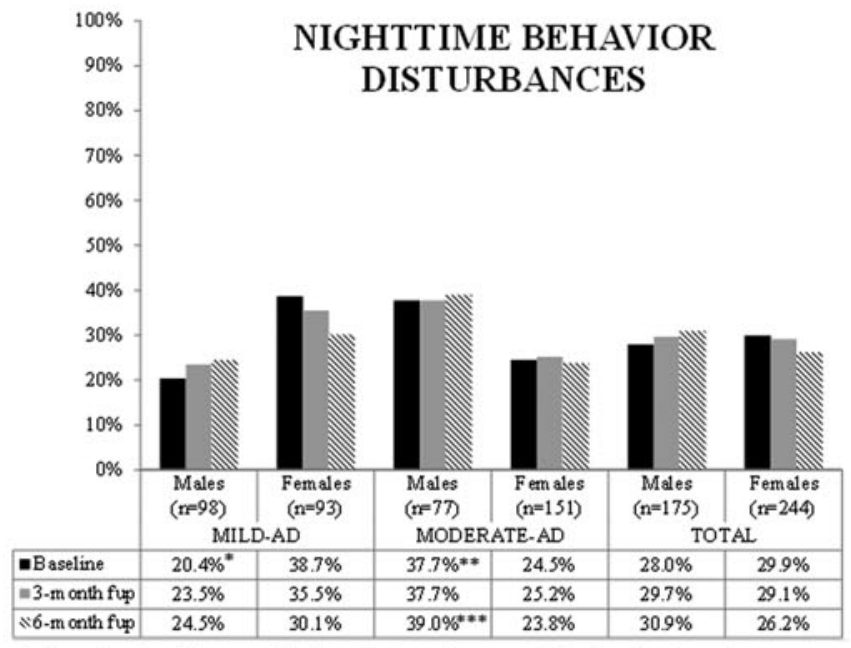

${ }^{*} X^{2} p$-value $(m$ v $f)=0.006 ;{ }^{* *} X X^{2} p$-value $(m$ vs $f)=0.038 ; * * X^{2} p$-value $(m$ vs $f)=0.017$
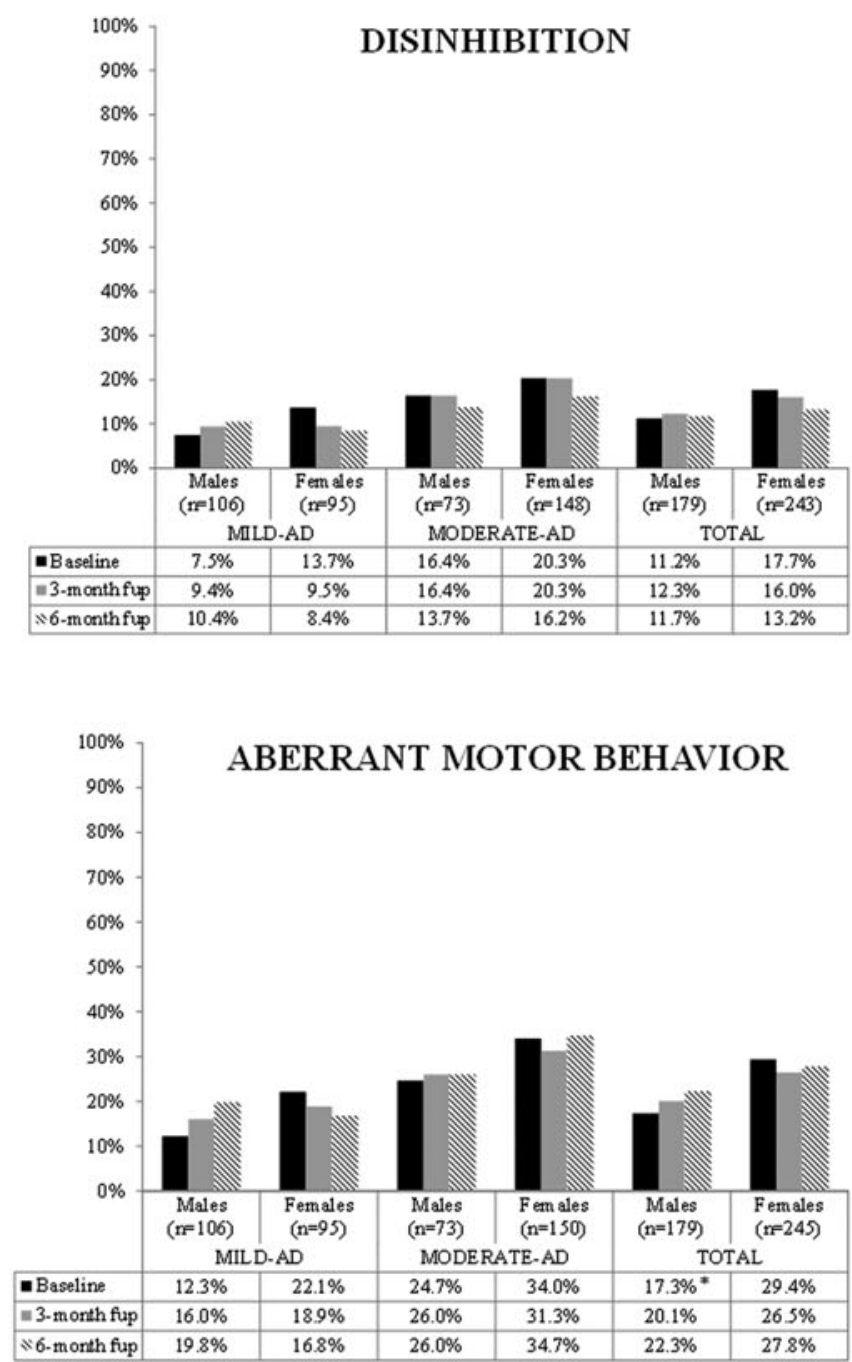

${ }^{*} X^{2} p$-value $(m$ vs $f)=0.004$.

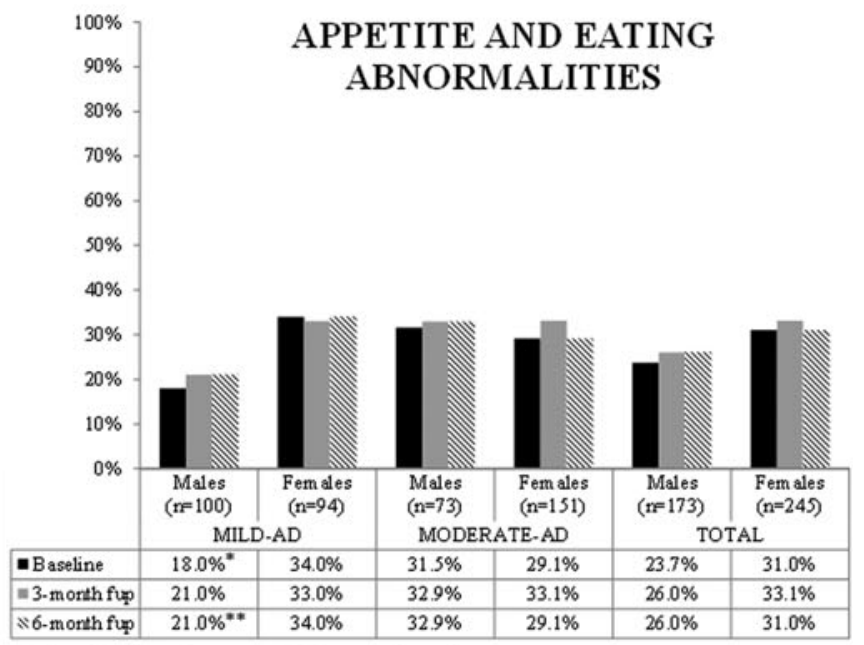

${ }^{*} X^{2} p$-value $(m$ vs $f)=0.011 . * X^{2} p$-value $(m v s f)=0.042$.

Not significant $(>0.05)$ p-values werenot reported.

FIG. 4. Frequency of neuropsychiatric symptoms according to NPI at study visits by gender-apathy, disinhibition, irritability, aberrant motor behavior, nighttime behavior disturbances and appetite and eating abnormalities. 
more frequent hallucinations, depression, anxiety, and apathy than men. At 6-month follow-up, the women suffered more than men from depression, apathy, appetite/eating abnormalities, and anxiety (see Figs. 3 and 4).

At 6-month follow-up, moderately demented men had more nighttime behavior disturbances than women (see Fig. 4).

\section{Association between gender and BPSD in mild- and moderate- $A D$ patients}

In most cases, the results of the exploratory analysis were confirmed by the multivariate logistic regressions (see Fig. 5).

After controlling for all the model variables, we found an association in mild-AD patients between gender and depression (odds ratio; OR [95\% confidence interval; $\mathrm{CI}] \mathrm{fe}-$ male vs. male $=3.32[1.44-7.67])$, anxiety $(\mathrm{OR}[95 \% \mathrm{CI}]$ female vs. male $=2.42[1.23-4.79])$, apathy $(\mathrm{OR}[95 \% \mathrm{CI}]$ female vs. male $=2.25$ [1.07-4.70]), nighttime behavior disturbances (OR [95\% CI] female vs. male $=3.97$ [1.66-9.49]), and appetite/eating abnormalities (OR [95\% CI] female vs. male $=2.39$ [1.10-5.18])

Moderate-AD female patients displayed euphoria more frequently than male patients (OR $[95 \% \mathrm{CI}]$ female vs. male $=3.67[1.25-10.74]$ ).

\section{Effect of time on BPSD in mild- and moderate- $A D$ patients}

In mild-AD patients, no significant effect of time emerged in any of the NPI domains; in this group of patients, the frequency of BPSD did not change significantly.

In moderate- $\mathrm{AD}$ patients, an association was found between time and delusions (OR [95\% CI] at 3-month followup vs. baseline $=0.79[0.64-0.98])$, anxiety $(\mathrm{OR}[95 \% \mathrm{CI}]$ visit at 3 months vs. baseline $=0.73[0.57-0.93]$ ), and irrita-

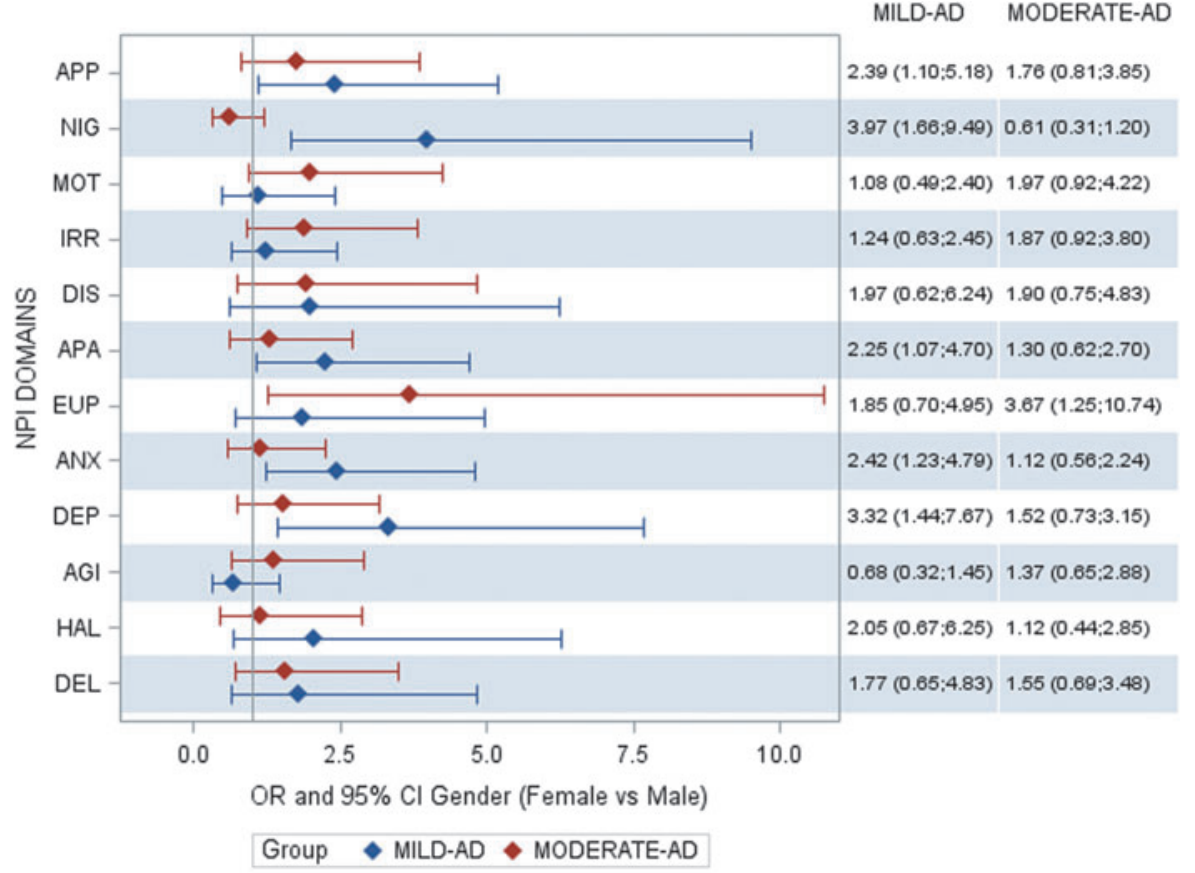

FIG. 5. Multivariate logistic models, BPSD, and gender (odds ratios females vs. males).

bility (OR $[95 \% \mathrm{CI}]$ visit at 3 months vs. baseline $=0.70$ [0.53-0.93]). The frequency of delusions, anxiety, and irritability decreased during the first 3 months after the switch, independently of gender (as noted above, gender was in fact not associated with any of these domains).

No significant associations emerged between time and ther BPSD.

These results (thanks to control of confounding factors through multivariate estimation) actually overcome those of our exploratory analysis about the frequency of patients with remitted NPI symptoms during follow-up (in the interests of providing fuller information, we reported results of exploratory analysis in Supplementary Table S1 (Supplementary Data are available online at www.liebertpub.com/jwh).

\section{Discussion}

As expected, ${ }^{32}$ women were overrepresented, and also somewhat more cognitively impaired than men, both at baseline and throughout the 6-month follow-up period. In our sample, women and men had their therapy switched for the same reasons, proportionately, mainly lack of response to treatment.

Our post hoc analysis highlights more potentially relevant gender differences in BPSD. Mild-AD women were more likely than men to experience BPSD, such as depression, anxiety, apathy, nighttime behavior disturbances, and appetite/eating abnormalities, while the frequency of BPSD did not change significantly over time in the 6 months after the switch. In the moderate-AD sample, gender differences on BPSD were flattened (except for euphoria) and the frequency of delusions, anxiety, and irritability decreased during the first 3 months after the switch, independently of gender.

The female predominance in behavior disturbances was also reflected in the use of psychoactive medications, as women were more likely than men to be taking antidepressants, but not

\section{MILD-AD MODERATE-AD}

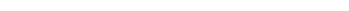

BPSD, Behavioral and Psychological Symptoms of Dementia. APP, appetite and eating abnormalities; AGI, agitation; ANX, anxiety; APA, apathy; DEL, delusions; DEP, depression; DIS, disinhibition; EUP, euphoria; HAL, hallucinations; IRR, irritability; MOT, aberrant motor behavior; NIG, nighttime behavior disturbances. 
antipsychotics. Our data are in line with previous studies that found women with $\mathrm{AD}$ more often displaying a broader spectrum of BPSD, ${ }^{33,34}$ and gender was found to be a strong predictor of behavioral differences among AD patients. ${ }^{12}$ Women with AD were shown to suffer delusions and hallucinations more often ${ }^{8,9}$ and earlier ${ }^{10}$ than men. Furthermore, depression was associated with twice as great a risk of $\mathrm{AD}$ in women but not in men, ${ }^{35}$ and depression itself was found to be more prevalent in women with AD. ${ }^{9,11}$

In other studies, men have been reported as more commonly suffering apathy, ${ }^{10,12}$ aggression, ${ }^{12,13}$ and vegetative changes such as overeating and oversleeping. ${ }^{12}$ Other studies of $\mathrm{AD}$ have found a higher, but not statistically significant, incidence of aggressive behavior in men. ${ }^{36,37}$ Our own data confirm these findings only in part, probably owing to differences in the samples and in the tools used to assess BPSD.

Our study has a number of strengths. The sample was larger than in most previous gender studies on AD, as subjects were recruited from a number of memory clinics across Italy. Sociodemographic and clinical data were collected using uniform structured evaluations. We used a descriptive statistical analysis (with exploratory $p$ values) supported by confirmatory multivariate models.

Our project assesses gender differences in BPSD profile after the switch from oral $\mathrm{ChEi}$ to transdermal rivastigmine patch. However, our work also has limitations. Our pooled data lack a control population. To reduce possible bias, the study considered only patients who had been switched from oral ChEi to rivastigmine patch therapy.

\section{Conclusions}

Our study, like most previous research on AD patient populations, indicates that the prevalence of various BPSD differs between women and men. Mild-AD women tend to experience BPSD more often than men; but where cognitive dysfunction is more severe, that is, among the moderate-AD patients, the prevalence of BPSD tends to be comparable between genders. Our data also suggest that in the 3 months after the switch, moderate-AD patients experienced a decrease in delusions, anxiety, and irritability, again with no substantial gender difference. The need is for substantially increased gender-specific data on treatment response, which could carry major prognostic and therapeutic implications in clinical practice.

\section{Acknowledgments}

The study was supported by a grant from Novartis Farma S.p.A., Origgio (VA), Italy. The authors meet the criteria of the International Committee of Medical Journal Editors (ICMJE) for authorship, and were fully responsible for all aspects of article development. They are grateful to Sara Rizzoli and Shalom Haggiag for help in drafting the article.

\section{Author Disclosure Statement}

D.C. is a part-time employee of Novartis Farma, Italy, and received grants from Allergan and Aventis. E.Z. is an employee of Novartis Farma, Italy. G.S. has been funded by the Italian Ministry of Health, FB-Health, and the European Commission. L.S. and A.O. are employees of MediNeos Observational Research. No other conflicts of interest in this work have been reported by the authors.

\section{References}

1. Devshi R, Shaw S, Elliott-King J, et al. Prevalence of behavioral and psychological symptoms of dementia in individuals with learning disabilities. Diagnostics (Basel) 2015; 5:564-576.

2. Van der Linde RM, Dening T, Matthews FE, Brayne C. Grouping of behavioral and psychological symptoms of dementia. Int J Geriatr Psychiatry 2013;29:563-567.

3. Hurt C, Bhattacharyya S, Burns A, et al. Patient and caregiver perspectives of quality of life in dementia. An investigation of the relationship to behavioral and psychological symptoms in dementia. Dement Geriatr Cogn Disord 2008;26:138-146.

4. Ornstein K, Gaugler JE, Devanand DP, Scarmeas N, Zhu C, Stern Y. The differential impact of unique behavioral and psychological symptoms for the dementia caregiver: How and why do patients' individual symptom clusters impact caregiver depressive symptoms? Am J Geriatr Psychiatry 2013;21:1277-1286.

5. Dubois B, Padovani A, Scheltens P, Rossi A, Dell'Agnello G. Timely diagnosis for Alzheimer's disease: A literature review on benefits and challenges. J Alzheimers Dis 2015; 49:617-631.

6. Borroni B, Costanzi C, Padovani A. Genetic susceptibility to behavioral and psychological symptoms in Alzheimer disease. Curr Alzheimer Res 2010;7:158-164.

7. Gardette V, Lapeyre-Mestre M, Coley N, et al. Antipsychotic use and mortality risk in community-dwelling Alzheimer's disease patients: Evidence for a role of dementia severity. Curr Alzheimer Res 2012;9:1106-1116.

8. Devanand DP, Brockington CD, Moody BJ, et al. Behavioral syndromes in Alzheimer's disease. Int Psychogeriatr 1992;4 Suppl 2:161-184.

9. Cohen D, Eisdorfer C, Gorelick P, et al. Sex differences in the psychiatric manifestations of Alzheimer's disease. J Am Geriatr Soc 1993;41:229-232.

10. Jost BC, Grossberg GT. The evolution of psychiatric symptoms in Alzheimer's disease: A natural history study. J Am Geriatr Soc 1996;44:1078-1081.

11. Lazarus LW, Newton N, Cohler B, Lesser J, Schweon C. Frequency and presentation of depressive symptoms in patients with primary degenerative dementia. Am J Psychiatry $1987 ; 144: 41-45$.

12. Ott BR. Cognition and behavior in patients with Alzheimer's disease. J Gend Specif Med 1999;2:63-69.

13. Eastley R, Wilcock GK. Prevalence and correlates of aggressive behaviors occurring in patients with Alzheimer's disease. Int J Geriatr Psychiatry 1997;12:484-487.

14. Pinto T, Lanctôt KL, Herrmann N. Revisiting the cholinergic hypothesis of behavioral and psychological symptoms in dementia of the Alzheimer's type. Ageing Res Rev 2011; 10:404-412.

15. Campbell N, Ayub A, Boustani MA, et al. Impact of cholinesterase inhibitors on behavioral and psychological symptoms of Alzheimer's disease: A meta-analysis. Clin Interv Aging 2008;3:719-728.

16. Winblad B, Grossberg G, Frölich L, et al. IDEAL: A 6month, double-blind, placebocontrolled study of the first skin patch for Alzheimer disease. Neurology 2007;69(4 Suppl 1):S14-S22.

17. Cumbo E, Ligori LD. Differential effects of current specific treatments on behavioral and psychological symptoms in patients with Alzheimer's disease: A 12-month, randomized, open-label trial. J Alzheimers Dis 2014;39:477-485. 
18. Norton MC, Clark C, Fauth EB, et al. Caregiver personality predicts rate of cognitive decline in a community sample of persons with Alzheimer's disease. The Cache County Dementia Progression Study. Int Psychogeriatr 2013;25:16291637.

19. Fauth E, Hess K, Piercy K, Norton M, et al. Caregivers' relationship closeness with the person with dementia predicts both positive and negative outcomes for caregivers' physical health and psychological well-being. Aging Ment Health 2012;16:699-711.

20. Spalletta G, Caltagirone C, Padovani A, et al. EVOLUTION study Working Group. Cognitive and affective changes in mild to moderate Alzheimer's disease patients undergoing switch of cholinesterase inhibitors: A 6-month observational study. PLoS One 2014;9:e89216.

21. Rocca WA, Mielke MM, Vemuri P, Miller VM. Sex and gender differences in the causes of dementia: A narrative review. Maturitas 2014;79:196-201.

22. Carter CL, Resnick EM, Mallampalli M, Kalbarczyk A. Sex and gender differences in Alzheimer's disease: Recommendations for future research. J Womens Health 2012;21:1018-1023.

23. Mielke MM, Vemuri P, Rocca WA. Clinical epidemiology of Alzheimer's disease: Assessing sex and gender differences. Clin Epidemiol 2014;6:37-48.

24. Colombo D, Bellia G, Vassellatti D, Zagni E, Sgarbi S, Rizzoli S. A gender-medicine post hoc analysis (Meta$\mathrm{GeM}$ ) project to test sex differences in previous observational studies in different diseases: Methodology. Open Access J Clin Trials 2014;6:111-116.

25. McKhann G, Drachman D, Folstein M, et al. Clinical diagnosis of Alzheimer's disease: Report of the NINCDSADRDA Work Group under the auspices of Department of Health and Human Services Task Force on Alzheimer's Disease. Neurology 1984;34:939-944.

26. Folstein MF, Folstein SE, McHugh PR. "Mini-mental state". A practical method for grading the cognitive state of patients for the clinician. J Psychiatr Res 1975;12:189-198.

27. Magni E, Binetti G, Bianchetti A, Rozzini R, Trabucchi M. Mini-Mental State Examination: A normative study in Italian elderly population. Eur J Neurol 1996;3:198-202.

28. Cummings JL, Mega M, Gray K, Rosenberg-Thompson S, Carusi DA, Gorbein J. The Neuropsychiatric Inventory: Comprehensive assessment of psychopathology in dementia. Neurology 1994;44:2308-2314.
29. Binetti G, Mega MS, Magni E, et al. Behavioral disorders in Alzheimer disease: A transcultural perspective. Arch Neurol 1998;55:539-544.

30. Lyketsos CG, Lopez O, Jones B, et al. Prevalence of neuropsychiatric symptoms in dementia and mild cognitive impairment: Results from the cardiovascular health study. JAMA 2002;288:1475-1483.

31. Steinberg M, Tschanz JT, Corcoran C, et al. The persistence of neuropsychiatric symptoms in dementia: The Cache County Study. Int J Geriatr Psychiatry 2004;19: 19-26.

32. Hebert LE, Scherr PA, McCann JJ, Beckett LA, Evans DA. Is the risk of developing Alzheimer's disease greater for women than for men? Am J Epidemiol 2001;153:132-136.

33. Kitamura T, Kitamura M, Hino S, Tanaka N, Kurata K. Gender differences in clinical manifestations and outcomes among hospitalized patients with behavioral and psychological symptoms of dementia. J Clin Psychiatry 2012;73: $1548-1554$.

34. Ono T, Tamai A, Takeuchi D, et al. Predictors of length of stay in a ward for demented elderly: Gender differences. Psychogeriatrics 2010;10:153-159.

35. Artero S, Ancelin ML, Portet F, et al. Risk profiles for mild cognitive impairment and progression to dementia are gender specific. J Neurol Neurosurg Psychiatry 2008;79: 979-984.

36. Drachman DA, Swearer JM, O’Donnell BF, Mitchell AL, Maloon A. The Caretaker Obstreperous-Behavior Rating Assessment (COBRA) Scale. J Am Geriatr Soc 1992;40: 463-470.

37. Ryden MB. Aggressive behavior in persons with dementia who live in the community. Alzheimer Dis Assoc Disord 1988;2:342-355.
Address correspondence to:

Emanuela Zagni, MSc

Patient Access

Novartis Farma S.p.A.

Largo Umberto Boccioni, 1

Origgio, VA 21040

Italy

E-mail: medidata@medineos.com 